\title{
Changes in skeletal-muscle myosin isoenzymes with hypertrophy and exercise
}

\author{
Patricia GREGORY,* Robert B. LOW and William S. STIREWALT $\dagger$ \\ Department of Physiology and Biophysics, University of Vermont, College of Medicine, Burlington, VT 05405, U.S.A.
}

\begin{abstract}
The patterns of myosin isoenzymes in fast- and slow-twitch muscles of the rat hindlimb were studied, by pyrophosphate/polyacrylamide-gel electrophoresis, with hypertrophy (induced by synergist removal) and with spontaneous running exercise of 4 and 11 weeks duration. At 11 weeks, changes with hypertrophy in the slow-twitch soleus, composed of $>95 \%$ SM2 (slow myosin 2) in normal muscles, were minor, and consisted of an increase in the SM1 and SM1', and a loss of intermediate myosin (IM), an isoenzyme characteristic of Type IIa fibres [Fitzsimons \& Hoh (1983) J. Physiol. (London) 343, 539-550]. The changes were dramatic, however, in the fast-twitch plantaris muscle. There was a 3-fold increase in the proportion of SM. In addition, IM became the predominant isoenzyme in the profile of hypertrophied plantaris by 4 weeks. These increases were balanced by decreases in the proportion of FM2 (fast myosin 2), with FM1 completely absent from the profile at 11 weeks. The changes in the plantaris with exercise were similar in direction but not as extensive as those with hypertrophy, and FM1 remained present at control levels throughout the study. When hypertrophy and exercise were combined, the increase in slow myosin was equal to the sum of the increases with each treatment alone. Changes at 4 weeks were intermediate between those of control and 11-week muscles. Peptide mapping of individual myosin isoenzymes showed that the heavy chains of IM were different from either fast or slow heavy chains. Furthermore, IM was found to be composed of a mixture of fast and slow light chains. These changes suggest that a transformation of myosin from fast to slow isoforms was in progress in the plantaris in response to hypertrophy, via a Type-IIa-myosin (IM) intermediate stage, a phenomenon similar to that occurring in chronically stimulated fast muscles during fast-to-slow transformation [Brown, Salmons \& Whalen (1983) J. Biol. Chem. 258, 14686-14692].
\end{abstract}

\section{INTRODUCTION}

The expression of myosin isoenzymes changes during muscle development (Hoh, 1979; Whalen et al., 1979), hormonal treatment (Chizzonite et al., 1982), altered contractile function (Litten et al., 1982), and disease states (Fitzsimons \& Hoh, 1983; Rutschmann et al., 1984). These isoenzyme changes appear to be the result of differential gene transcription (Weydert et al., 1983; Everett et al., 1984; Izumo et al., 1986). Thus the myosin phenotype is not a fixed characteristic of a muscle, but exhibits a plasticity similar to other contractile proteins and metabolic enzymes.

Alteration of the neural input to a muscle, through cross-reinnervation of fast and slow muscles or by the imposition of artificial electrical stimulation of a fast-twitch muscle, leads to changes in the myosin isoenzyme profile (Hoh et al., 1980; Mabuchi et al., 1982). Thus the type of motoneuron innervation, pattern of stimulation or resultant muscle activity are also important determinants of the expression of myosin isoforms.

In the present study, we have observed the effects of work-overload hypertrophy and running exercise on the myosin isoenzyme composition of rat fast- and slowtwitch muscles in order to determine whether functional alterations that leave the existing nerve supply intact can produce similar changes. We have found dramatic alterations in the myosin isoenzyme profile of the fast-twitch plantaris muscle, and minor alterations in the slow-twitch soleus muscle, with both treatments. In addition to a large increase in slow myosin (SM), we found that the predominant isoenzyme induced in the plantaris is intermediate myosin (IM), which we further show is composed of unique heavy chains and a combination of fast and slow light-chain types. The results of these studies have been reported in a preliminary, abstract, form (Reilly et al., 1984; Gregory et al., 1985).

\section{METHODS}

Hypertrophy of the plantaris and soleus muscles was produced in 30 female Sprague-Dawley rats weighing $115-150 \mathrm{~g}$ by surgical removal of the synergist gastrocnemius muscle from both legs, under sodium methohexital (Brevital) anaesthesia. The surgical procedure was essentially as described by Baldwin et al. (1982), but in our experiments the soleus was not removed. Separate animals matched for body weight served as controls. A group of control animals was killed at the beginning of the study to determine whether there were any age-related changes in myosin isoenzymes during the course of the study. The animals were housed in groups of four on a

Abbreviations used: SM, slow myosin; IM, intermediate myosin; FM, fast myosin; LC $_{\mathrm{s}}$, slow-myosin light chain; LC, fast-myosin light chain; Bis, $N N^{\prime}$-methylenebisacrylamide.

* Present address: Department of Medicine, University of Chicago, 950 East 59th Street, Chicago, IL 60637, U.S.A.

$\dagger$ To whom reprint requests should be sent.

Vol. 238 


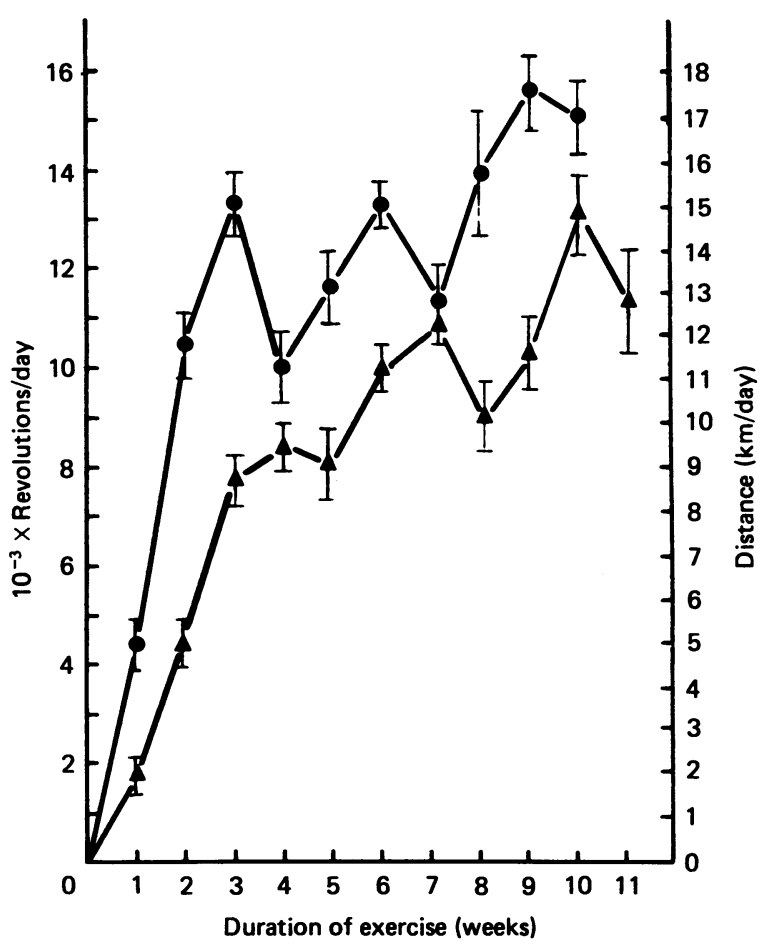

Fig. 1. Spontaneous exercise record

Exercised animals were housed in wheels where they ran voluntarily. Values are means \pm S.E.M. of average daily distances computed from weekly records of unoperated $(O)$ and operated $(\boldsymbol{\Delta})$ animals.

$12 \mathrm{~h}$ light $/ 12 \mathrm{~h}$ dark cycle and allowed access to food and water ad libitum.

Half of the operated and half of the control animals were placed in exercise wheels modified from those described by Mondon et al. (1980), in which they ran voluntarily. The animals lived in the wheel continuously and had access to food and water without leaving it. Records of the number of revolutions showed that the mean distance run increased steeply for the first 2 weeks and thereafter remained between 10000 and $16000 \mathrm{rev} . /$ day (Fig. 1). The circumference of the exercise wheel was $112 \mathrm{~cm}$; hence 895 revolutions of the wheel was equal to a distance of $1 \mathrm{~km}$. Animals that did not run at least $8 \mathrm{~km}$ per day by 4 weeks were excluded from the study. The operated animals did not run as well as unoperated animals, especially during the first week following surgery. We therefore killed the group of unoperated exercised animals after only 10 weeks of running in order to approximate more closely the training period of the operated animals. The operated animals maintained an exercise level of about $75-80 \%$ of the control level during the remainder of the study (Fig. 1).

The animals were killed 4 weeks and 11 weeks after surgery by cervical dislocation under Brevital anaesthesia. The hindlimbs were removed, skinned and immediately placed on ice. The soleus and plantaris muscles were dissected, frozen in liquid $\mathrm{N}_{2}$, weighed and stored at $-70^{\circ} \mathrm{C}$ for later analyses.

Methods for separation of myosin isoenzymes by pyrophosphate/polyacrylamide-gel electrophoresis were essentially as described by Hoh et al. (1976). The gels were stained overnight with $0.025 \%$ Coomassie Brilliant Blue R-250/25\% (v/v) propan-2-ol/10\% (v/v) acetic acid, and destained in $10 \%$ acetic acid. Relative amounts of each isoenzyme were estimated by scanning duplicate gels with a high-resolution laser-beam densitometer and integrating the areas under the peaks (Brayden \& Halpern, 1983). Reproducibility between duplicate gels was approx. $\pm 2 \%$. Although absolute quantification of isoenzymes is difficult, changes in their relative amounts could be assessed with a reasonable degree of accuracy.

Samples of purified myosin isoenzymes for analysis of heavy- and light-chain composition were obtained by slicing individual bands from the pyrophosphate gels after staining for $15 \mathrm{~min}$ in $0.05 \%$ Coomassie Brilliant Blue $/ 50 \%$ (v/v) methanol $10 \%$ acetic acid, and destaining in cold distilled water. We chose the bands to be used for peptide-mapping studies carefully in order to minimize contamination with isoenzymes in adjacent bands. For the sample of SM we used the SM2 band from gels of normal soleus (Fig. $3 a$ below). IM was obtained from hypertrophied/exercised plantaris, because the IM band of these gels was most clearly separated from the fast myosin bands (Fig. $2 d$ below), and could be isolated in the largest quantities from these muscles. The sample of FM was either FM1 or FM2 from normal plantaris, since these bands are not adjacent to IM (Fig. $2 a$ below). Myosin light chains are still present in these isoenzyme slices. Whalen et al. (1979) have shown, however, that the pattern of heavy-chain cleavage by Staphylococcus aureus V8 proteinase is not affected by the presence of light chains.

Peptide mapping was performed as described by Cleveland et al. (1977) on three or four pyrophosphate gel slices, each containing approx. $1 \mu \mathrm{g}$ of myosin of a single isoenzyme type. The separating gel was $12 \mathrm{~cm}$ long and made up of $12 \%$ acrylamide (acrylamide/Bis, 200:1, w/w; Bio-Rad), $0.1 \%$ SDS, $10 \%$ (v/v) glycerol and 0.75 м-Tris/HCl, pH 9.3 (Thomas, 1978). Staphylococcus aureus V8 proteinase (Miles Laboratories) was added to each well in either $0.01 \mu \mathrm{g}$ or $0.1 \mu \mathrm{g}$ amounts. Digestion took place in the stacking gel during an overnight run at $5 \mathrm{~mA} /$ plate. The run was completed the next morning at $18 \mathrm{~mA} /$ plate, and the gels removed after the Bromophenol Blue dye front ran off. After fixation in $50 \%$ methanol $/ 10 \%$ acetic acid, and cross-linking overnight in $10 \%(\mathrm{v} / \mathrm{v})$ glutaraldehyde (Kodak), the gels were silver-stained by the method of Giulian et al. (1983).

Myosin light chains were analysed in the same gels as those used for peptide mapping, except that the V8 proteinase was omitted from the wells.

Differences between groups were evaluated by analysis of variance (Snedecor \& Cochran, 1968). In cases of inhomogeneity of variances, a Kruskal-Wallis one-way analysis of variance was used (Hollander \& Wolfe, 1973). A probability of less than 0.05 was considered statistically significant.

\section{RESULTS}

\section{Muscle hypertrophy}

Mean body weights, muscle weights and muscle-weightto-body-weight ratios of all treatment groups are listed in Table 1. The rate of increase in body weight and muscle weight attributable to normal growth was established by the control group. At 11 weeks the body 
Table 1. Muscle wet weights and body weights

Values are expressed as means \pm S.E.M. Numbers in parentheses indicate number of muscles studied.

\begin{tabular}{|c|c|c|c|c|c|c|}
\hline Treatment & $\begin{array}{c}\text { Duration } \\
\text { (weeks) }\end{array}$ & $\begin{array}{l}\text { Body } \\
\text { wt. (g) }\end{array}$ & $\begin{array}{l}\text { Plantaris } \\
\text { wt. (mg) }\end{array}$ & 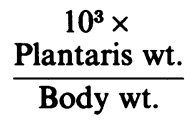 & $\begin{array}{c}\text { Soleus } \\
\text { wt. (mg) }\end{array}$ & $\begin{array}{c}10^{3} \times \\
\text { Soleus wt. } \\
\text { Body wt. }\end{array}$ \\
\hline Control & $\begin{array}{r}0 \\
4 \\
11\end{array}$ & $\begin{array}{l}140 \pm 2 \\
198 \pm 9 \\
250 \pm 4\end{array}$ & $\begin{array}{ll}133 \pm 3 & (12) \\
212 \pm 9 & (12) \\
273 \pm 3 & (18)\end{array}$ & $\begin{array}{l}0.95 \pm 0.02 \\
1.07 \pm 0.02 \\
1.11 \pm 0.01\end{array}$ & $\begin{array}{r}61 \pm 2(12) \\
82 \pm 4(12) \\
104 \pm 3(11)\end{array}$ & $\begin{array}{l}0.44 \pm 0.01 \\
0.41 \pm 0.01 \\
0.42 \pm 0.01\end{array}$ \\
\hline Hypertrophy & $\begin{array}{r}4 \\
11\end{array}$ & $\begin{array}{l}196 \pm 7 \\
261 \pm 6\end{array}$ & $\begin{array}{l}354 \pm 9(14) \\
427 \pm 19(16)\end{array}$ & $\begin{array}{l}1.84 \pm 0.08 \\
1.63 \pm 0.05\end{array}$ & $\begin{array}{l}118 \pm 6(11) \\
123 \pm 7(11)\end{array}$ & $\begin{array}{l}0.58 \pm 0.03 \\
0.48 \pm 0.02\end{array}$ \\
\hline Exercise & $\begin{array}{r}2 \\
4 \\
10\end{array}$ & $\begin{array}{l}147 \pm 0 \\
197 \pm 5 \\
225 \pm 4\end{array}$ & $\begin{array}{l}149 \pm 3 \\
205 \pm 8 \\
246 \pm 11\end{array}$ & $\begin{array}{l}1.01 \pm 0.02 \\
1.04 \pm 0.03 \\
1.10 \pm 0.06\end{array}$ & $\begin{array}{r}58 \pm 5(4) \\
96 \pm 4(10) \\
127 \pm 3(8)\end{array}$ & $\begin{array}{l}0.39 \pm 0.04 \\
0.49 \pm 0.02 \\
0.57 \pm 0.02\end{array}$ \\
\hline $\begin{array}{l}\text { Hypertrophy } \\
\text { +exercise }\end{array}$ & $\begin{array}{r}4 \\
11\end{array}$ & $\begin{array}{l}183 \pm 5 \\
222 \pm 6\end{array}$ & $\begin{array}{l}357 \pm 10 \\
373 \pm 12\end{array}$ & $\begin{array}{l}1.96 \pm 0.06 \\
1.69 \pm 0.07\end{array}$ & $\begin{array}{l}122 \pm 5(11) \\
148 \pm 7\end{array}$ & $\begin{array}{l}0.66 \pm 0.03 \\
0.66 \pm 0.03\end{array}$ \\
\hline
\end{tabular}

weights of spontaneously exercised animals were significantly less than non-exercised animals $(P<0.01)$, an observation that has been attributed to decreased adiposity (Mondon et al., 1980). Thus it was important to consider both absolute and body-weight-normalized muscle weights to appreciate the nature and significance of the changes that occurred.

The absolute weight of the hypertrophied plantaris was increased over control values by $67 \%$ at 4 weeks and by $56 \%$ at 11 weeks $(P<0.001)$. Normalized muscle weights were increased over controls by $72 \%$ and $47 \%$ respectively $(P<0.001)$.

The absolute weight of the soleus was increased over control values by $43 \%$ at 4 weeks and by $18 \%$ at 11 weeks $(P<0.05)$. Normalized weights were increased over control by $42 \%$ at 4 weeks but only by $13 \%$ at 11 weeks $(P<0.05)$.

Exercise alone did not affect the absolute or normalized weight of the plantaris in either the presence or absence of hypertrophic stress. In contrast, the absolute weight of the soleus were increased $22 \%$ by exercise alone at 10 weeks $(P<0.001)$. Absolute soleus weights in the hypertrophy-plus-exercise group were significantly increased at both 4 weeks $(49 \%)$ and 10 weeks $(43 \%)$. Normalized soleus weights were increased with exercise alone by $18 \%$ at week 4 and by $34 \%$ at week $11(P<0.001)$. These values were increased still further in the hypertrophy-plus-exercise group, the effect being approximately equal $(60 \%)$ at both time points.

\section{Changes in myosin isoenzymes in plantaris}

Five isoenzyme bands were present on the gels of every control plantaris (Fig. 2 and Table 2). The bands were identified by co-electrophoresis with extracts of muscles whose isoenzyme composition has previously been described in the literature. The first four bands co-migrated with the fast (FM) and intermediate (IM) isoenzymes of the extensor digitorum longus (Hoh et al., 1980) and accordingly were labelled FM1, FM2, FM3, and IM in order of decreasing mobility (Fitzsimons \& Hoh, 1983; Hoh \& Chow, 1983). IM is an isoenzyme associated with fast Type-IIa fibres. A small amount of SM was also present, amounting to about $5 \%$ of the total. This SM co-migrated with the major isoenzyme of soleus and was therefore labelled SM2 (Maréchal et al., 1984). This analysis agrees well with the fibre-type composition of the rat plantaris of $53 \%$ fast-oxidative-glycolytic (IIa), $41 \%$ fast-glycolytic (IIb), and $6 \%$ slow-oxidative (I) fibres (Ariano et al., 1973).

A number of striking changes occurred in the isoenzyme profile of the hypertrophied plantaris (Table 2). The mean percentage of SM was nearly doubled by 4 weeks $(P<0.001)$, and was on average more than three times the control level at 11 weeks $(P<0.001)$. A second species of SM appeared with hypertrophy (Fig. $2 b$ ) that migrated just ahead of the major species (SM2), and was referred to as SM1, in keeping with current conventions (Hoh, 1978; Lyons et al., 1983; Maréchal et al., 1984).

Hypertrophy of the plantaris also resulted in a dramatic increase in IM, which became the predominant isoenzyme in hypertrophied muscles within 4 weeks, making up about $40 \%$ of the total myosin in hypertrophied muscles $(P<0.001)$. This isoenzyme appeared to have a slightly lower mobility than that of the IM of control plantaris, as it separated better from FM3 in the gel (Figs. $2 a$ and $2 b$ ).

The increases in SM and IM in hypertrophied plantaris were accompanied by losses in FM1 and, to a lesser extent, FM2, whereas the proportion of FM3 remained relatively constant throughout the study. The percentage of FM1 was reduced by half at 4 weeks and it completely disappeared from the profile at 11 weeks.

Although our measurements only indicate relative increases in SM and IM, they must also represent absolute increases, since hypertrophied muscles were $50-70 \%$ larger than controls. Hypertrophied and control plantaris have been shown to contain the same amount of myosin per unit weight (Baldwin et al., 1982).

The isoenzyme changes in the plantaris with running exercise were similar to, but not identical with, the changes with hypertrophy. The mean percentage of SM was approximately doubled by 4 weeks $(P<0.001)$, and did not increase further by 10 weeks. Most of the exercised muscles also showed the appearance of SM1. IM was increased at 10 weeks $(P<0.01)$, but the increase was less pronounced than in hypertrophied muscles. The increases in SM and IM were balanced by losses of FM3 and FM2. However, FM1 persisted at control levels in exercised plantaris, in striking contrast with the situation in hypertrophy.

We examined a group of four muscles after only 2 weeks of exercise in order to define a time point where 


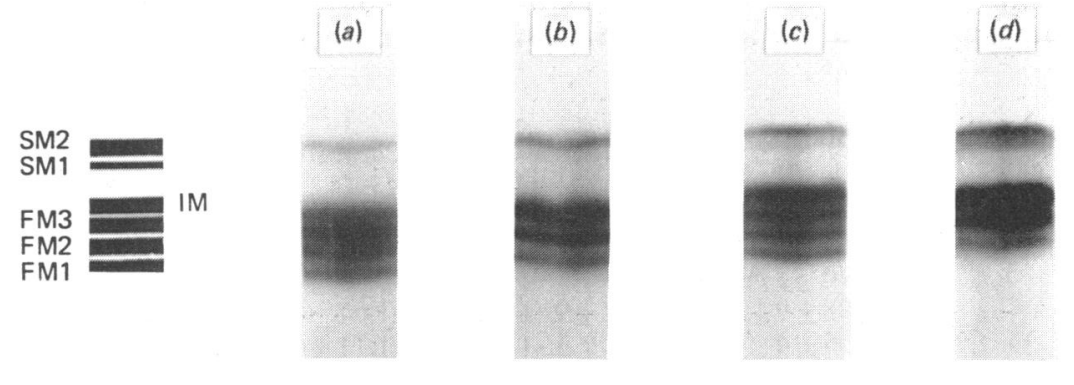

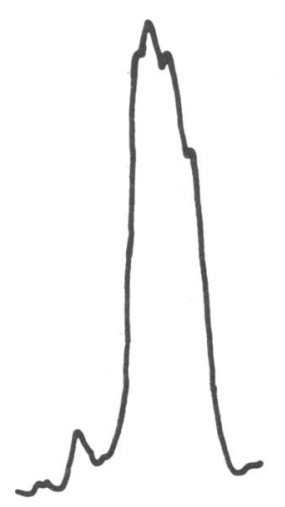

(a)

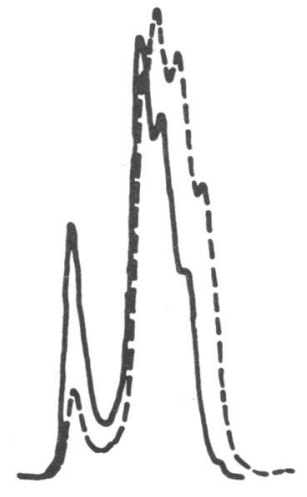

(b)

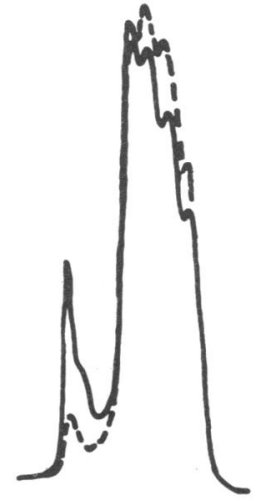

(c)

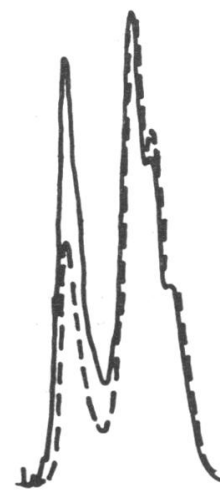

(d)

Fig. 2. Electrophoresis of native myosin isoenzymes of the plantaris

Pyrophosphate/polyacrylamide-gel electrophoresis was carried out essentially as described by Hoh et al. (1976). Typical gels are shown from $(a)$ normal, $(b)$ hypertrophy, $(c)$ exercise and $(d)$ combined-hypertrophy-and-exercise groups at 11 weeks. The bands are identified in the scheme on the left, the nomenclature of Fitzsimons \& Hoh (1983) being used. Densitometric scans of experimental groups $(b)$ and $(c)$ are superimposed on scans of normal plantaris. Scan (d) (hypertrophy plus exercise) is superimposed on scan (b) (hypertrophy) to illustrate alternations in the myosin isoenzyme profile.

Table 2. Percentage composition of myosin isoenzymes in plantaris

Values are expressed as means \pm S.E.M.

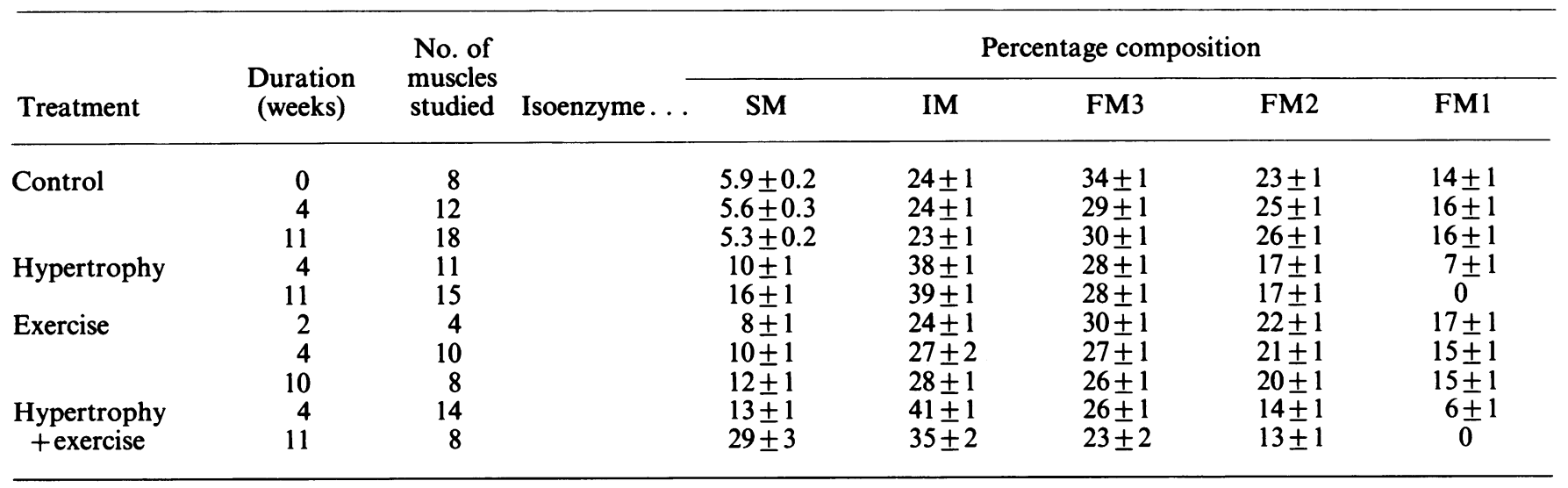

the isoenzyme changes with running exercise were still in transition. The level of SM was about halfway between control and 4-week levels, at about $8 \%$ of the total myosin. The proportion of IM was not significantly increased at 2 weeks. However, the species of IM found at 2 weeks was the slightly-slower-migrating form of IM mentioned above, which was seen in all hypertrophied and all exercised muscles.

The addition of running exercise to the hypertrophy process resulted in an increase in SM that was significantly greater than that of the hypertrophy group at 11 weeks $(P<0.001)$. The accumulation of SM was 


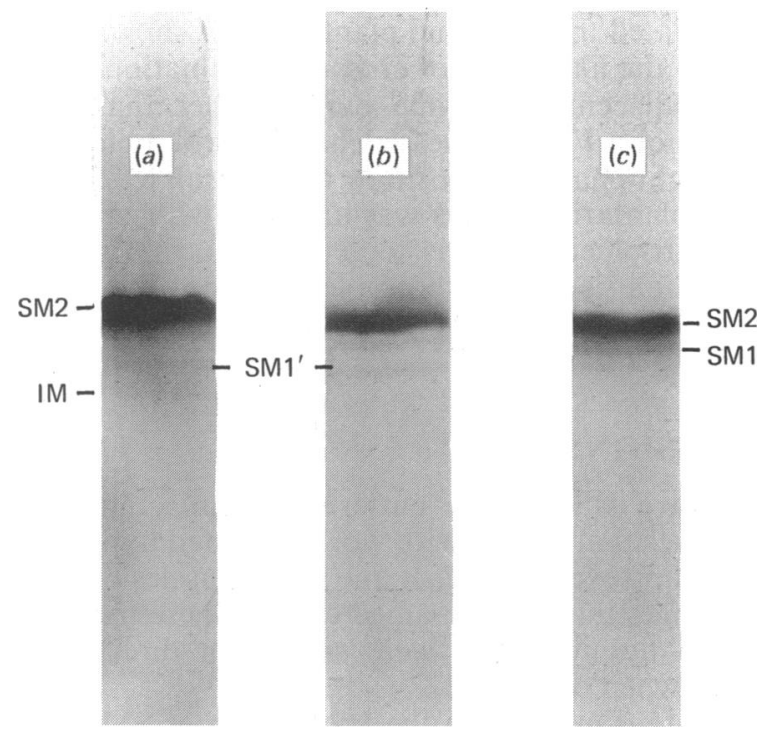

Fig. 3. Myosin isoenzyme changes in soleus

Pyrophosphate/polyacrylamide gels of soleus from (a) normal, (b) hypertrophy and (c) hypertrophy-plus-exercise groups at 11 weeks. Bands are identified according to the nomenclature of Marechal et al. (1984).

equal to the sum of the increases found with hypertrophy and exercise alone, reaching a mean level at 11 weeks, which was five times that of controls, and as high as seven times the control level in some individual muscles. The SM1 band was most prominent in the gels of the combined-hypertrophy-and-exercise plantaris when compared with either treatment alone. At the same time, the increase in IM we found with hypertrophy was not enhanced by the addition of exercise and appeared to have reached a maximum level similar to that of hypertrophy alone. The level of FM2 was reduced further than with hypertrophy alone at 11 weeks $(P<0.01)$. The muscles still retained a small portion of FM1 at 4 weeks, but contained no detectable FM1 at 11 weeks.

These alterations in myosin isoenzymes cannot be attributed to age-related changes, since we found the relative amounts of isoenzymes in the plantaris of animals killed at the beginning of the study to be nearly identical with those in control muscles at 4 weeks and 11 weeks (Table 2).

\section{Changes in myosin isoenzymes in soleus}

The results of our analysis of myosin isoenzymes in normal soleus (Fig. 3) are consistent with the fibre-type composition of this muscle, which contains primarily slow-twitch fibres as well as a small population (6-20\%) of fast Type-IIa fibres (Close, 1972). Thus the control soleus (Fig. 3a) was found to be composed of more than $95 \%$ SM2, with several minor bands, SM1, IM, and a third band whose position was in between IM and SM1 and which we refer to as SM1' according to the terminology used by Maréchal et al. (1984). These faintly stained minor bands could not be reproduced well photographically or quantified accurately by scanning in either the control or experimental groups. In addition to normally loaded gels, we also studied overloaded gels (results not shown) in order to increase the intensity of the minor bands.
Changes in the isoenzyme profile of the soleus with hypertrophy and exercise were much less pronounced than those in the plantaris and appeared to be limited to the three minor bands. The changes with hypertrophy (Fig. $3 b$ ) consisted of a disappearance of IM and an increase in the intensity of the SM1 and SM1' bands. The isoenzyme changes with running exercise (not shown) were characterized by an increase in SM1, a disappearance of IM, and a decrease in SM1' (which could still be seen faintly on overloaded gels). The combination of hypertrophy and exercise was characterized by a stronger increase in the intensity of SM1 than with exercise alone, and a disappearance of IM and SM1' (Fig. 3c). Again, these isoenzyme changes could only be evaluated qualitatively by visual inspection because they were limited to a small percentage of the total myosin. Nonetheless, the changes were observed consistently in all the muscles within a group.

\section{Heavy-chain and light-chain composition of IM}

We found that the peptide maps of FM and IM were similar, but that there were unique peptide fragments of

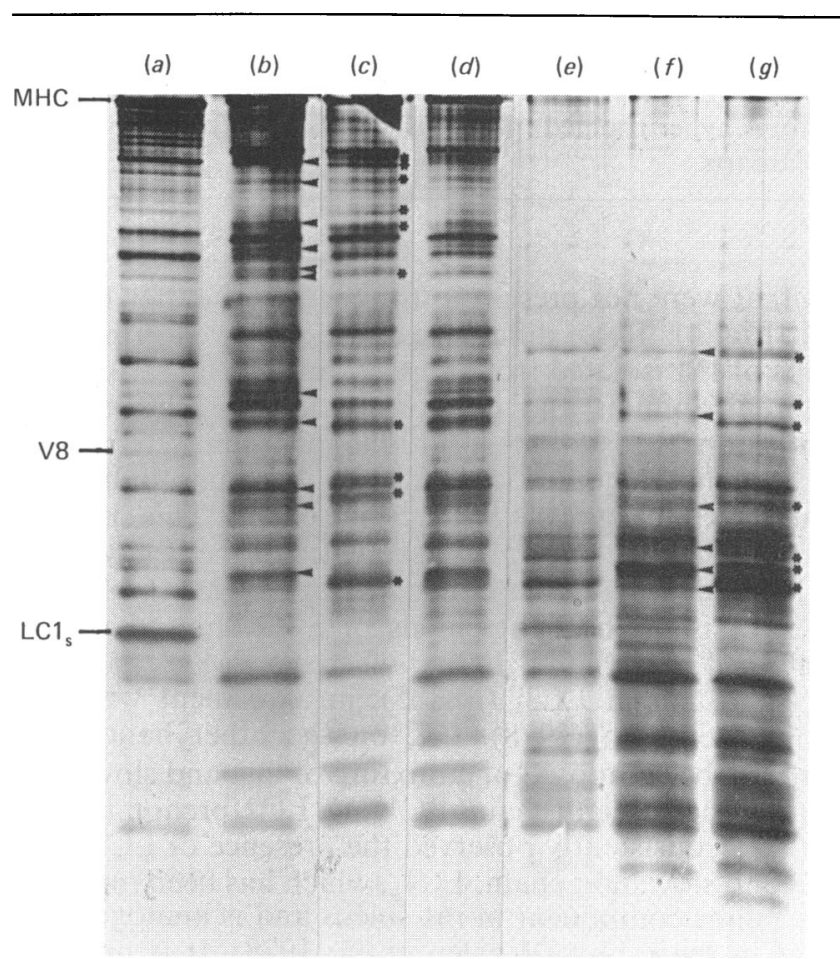

Fig. 4. Peptide mapping of individual myosin isoenzymes

Native myosin isoenzymes were isolated by slicing individual bands from pyrophosphate/polyacrylamide gels and digested with $0.01 \mu \mathrm{g}(a-d)$ or $0.1 \mu \mathrm{g}(e-g)$ of $S$. aureus V8 proteinase. Bands were revealed by silverstaining. Lanes $(e)-(g)$ represent an experiment separate from that of lanes $(a)-(d)$. (a) SM2 from normal soleus; $(b$ and $f$ ) IM from hypertrophied plantaris; ( $c$ and $g$ ) FM2 from normal plantaris; (d) IM from normal plantaris. 'MHC' indicates the position of myosin heavy chains, ' $\mathrm{V} 8$ ' the position of the $S$. aureus proteinase, and ' $\mathrm{LCl}_{\mathrm{s}}$ ' the position of the slowest-migrating light chain. Peptides appearing above this point in the gel are assumed to be cleavage products of the heavy chains. Differences between peptide maps of FM and IM are indicated by arrowheads ( $\longrightarrow$ ) (unique to IM) and asterisks $\left({ }^{*}\right)$ (unique to FM). 


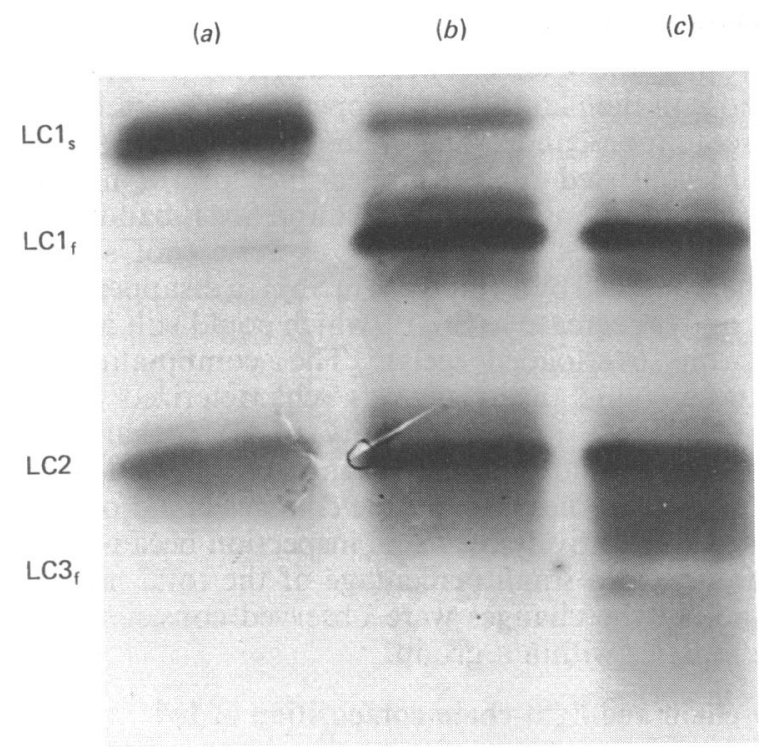

Fig. 5. Light-chain composition of individual myosin isoenzymes

Light chains were analysed in the same gels as those used in peptide-mapping experiments. Only the lower portion of the gel is shown. (a) SM2 from normal soleus; (b) IM from hypertrophied plantaris; (c) FM2 from normal plantaris.

IM that were not present in the digests of either FM or SM (Fig. 4). These differences were observed in peptide maps of IM isolated from hypertrophied plantaris of six different animals and digested with two enzyme concentrations. Most of these 'unique' peptides were present in the region of higher $M_{\mathrm{r}}$ than the light chains, indicating that they are fragments of the heavy chains. This suggests that the primary amino acid sequence of the heavy chain of IM is different from either FM or SM heavy chains.

Analysis of the light chains of SM2 showed that it contained $\mathrm{LC}_{\mathrm{s}}$ and $\mathrm{LC}_{\mathrm{s}}$, whereas $\mathrm{FM} 2$ contained $\mathrm{LC1}_{\mathrm{f}}, \mathrm{LC}_{\mathrm{f}}$ and $\mathrm{LC}_{\mathrm{p}}$ (Fig. 5), in agreement with the findings of Hoh (1978). IM, on the other hand, was found to be composed of a mixture of fast and slow light chains. The fast light chains $\mathrm{LCl}_{\mathrm{f}}$ and $\mathrm{LC} 2_{\mathrm{f}}$ predominated. We also consistently observed the presence of $\mathrm{LCl}_{\mathrm{s}}$ and a second slow light chain, $\mathrm{LCl}_{\mathrm{s}}$, which has been reported as a minor component in rat soleus and is analogous to $\mathrm{LC}_{\mathrm{S}}^{\mathrm{a}}$ in the rabbit (Whalen et al., 1978). It is unlikely that the slow light chains we found associated with IM were a result of contamination by SM, because SM migrated much slower than IM on the pyrophosphate gels from which these samples of IM were isolated. Any contamination of IM would more likely result from the nearby FM3 band, which would tend to exaggerate the predominance of fast light chains in IM.

The heavy-chain and light-chain analyses described above were performed on samples of IM isolated from hypertrophied muscles, because IM is more clearly separated from FM3 in those muscles. We also analysed the subunit composition of IM isolated from control plantaris in an attempt to determine why it migrated slightly faster on pyrophosphate gels than did IM from hypertrophied plantaris. The peptide map in this case contained peptide fragments characteristic of FM as well as IM (Fig. 4d). However, we also found this pattern for
FM3 isolated from normal plantaris (not shown), which points to the likelihood of cross-contamination by these closely adjacent isoenzyme bands rather than to the existence of a FM/IM heavy-chain hybrid molecule.

The light-chain composition of IM isolated from six control plantaris muscles was identical with that of IM of hypertrophied plantaris, indicating that the light-chain composition is not responsible for the difference in migration velocity between IM of normal and hypertrophied plantaris.

\section{DISCUSSION}

We have studied the pattern of change in myosin isoenzymes that occurs with work-overload hypertrophy and running exercise in fast and slow muscles of the rat. The changes that accompanied each of these treatments, although not identical, were similar in direction and additive when combined.

The 3-fold increase in the proportion of SM that we observed in hypertrophied plantaris suggests that a fast-to-slow fibre transformation may have occurred in some of the fibres. Indeed, the increase in SM that we found was of the same magnitude as the increase in the number of histochemically identifiable slow fibres with hypertrophy reported by others (Guth \& Yellin, 1971; Ianuzzo et al., 1976; Baldwin et al., 1982).

Several other possible explanations could account for this change. It is unlikely that new slow fibres were added to the muscle, in light of the conclusive study of Gollnick et al. (1981), who demonstrated no change in fibre number with hypertrophy by direct counts of individual dissected plantaris fibres. However, given the extreme change in workload in the synergist-removal model, it is possible that some fibre splitting may have occurred (Hall-Craggs, 1972), or that existing fast fibres were degraded and replaced by new slow fibres. Gambke $e t$ al. (1985) have recently presented evidence that the latter may occur in stimulation-induced fast-to-slow transformation.

Another possibility is that the increase in SM is accounted for by a greater relative hypertrophy of existing slow-twitch muscle fibres. Although a disproportionate increase in the cross-sectional area of slow fibres has been reported (Guth \& Yellin, 1971; Ianuzzo et al., 1976), those authors also reported an increase in the number of slow fibres per cross-section. Furthermore, slow fibres would have to enlarge to 14 times their normal size to account for the observed 7-fold increase in SM and near doubling in muscle size that we found in some individual muscles of the combined-hypertrophyand-exercise group. Such giant slow fibres have not been described by others.

In addition to the increase in SM, the predominance of IM in hypertrophied plantaris supports the interpretation of a fast-to-slow transformation in progress, since fibre transformation appears to proceed in a Type $\mathrm{IIb} \rightarrow \mathrm{IIa} \rightarrow \mathrm{I}$ sequence (Pierbon-Bormioli et al., 1981). Thus Brown et al. (1983) found that the proteolytic peptide map of myosin isolated from stimulated fast muscles undergoing transformation to slow initially lost peptides characteristic of Type-IIb myosin and gained peptides characteristic of Type-IIa myosin, whereas the Type-I-myosin pattern appeared only after longer periods of stimulation. If low-frequency stimulation is limited to only $8 \mathrm{~h} / \mathrm{day}$, the result is a conversion of 
myosin from the Type-IIb into the Type-IIa pattern on pyrophosphate gels, rather than the complete conversion into slow Type-F fibres that occurs with continuous stimulation (Mabuchi et al., 1982). IM is characteristic of Type-IIa fibres, and the proportion of IM in the isoenzyme profile of a muscle varies in relation to the extent of Type-IIa-fibre predominance (Hoh et al., 1980; Jolesz et al., 1981; Hoh \& Chow, 1983). The level of IM never rose above a maximum of $40-50 \%$ of the total myosin in the present study. Subsequent changes after IM had reached this level consisted of increases in SM with concomitant decreases in FM1 and FM2, suggesting that IM must be converted into SM before further FM-into-IM conversion occurs.

The proportion of histochemically identified Type-IIa fibres has been found not to increase in hypertrophied plantaris (Ianuzzo et al., 1981). However, histochemical fibre typing does not discriminate between the two subtypes of FM, probably because of the similarity in ATPase activity between FM and IM (Fitzsimons \& Hoh, 1983).

Slow motor units of the plantaris may experience greater usage during this model of hypertrophy, because the plantaris assumes a greater role in supporting body weight after removal of synergists. Whether existing fast motor units are converted into slow during hypertrophy, however, is not clear. Walsh et al. (1978) found no alterations in motor-unit types or in the percentage of each type in the motor-unit pool of hypertrophied medial gastrocnemius in the cat. However, these two models may not be comparable, since Walsh et al. (1978) also found no evidence of histochemical fibre-type conversion, the usual finding in hypertrophied rat plantaris.

Complete fast-to-slow fibre-type transformations have thus far been demonstrated only in cases where neural signals have been experimentally manipulated by crossinnervation or chronic low-frequency stimulation (Salmons \& Sreter, 1976; Hoh et al., 1980). Evidence is accumulating to suggest that such transformations can take place under more physiological conditions such as hypertrophy or exercise, when the nerve is left intact and the pattern of stimulation is not externally imposed (Baldwin et al., 1982; Roy et al., 1982; Noble et al., 1983; Green et al., 1984). Furthermore, it appears that a slow-type stimulation pattern is not critical for this transformation. Thus Sreter et al. (1982), using a phasic high-frequency stimulation pattern, found a replacement of fast isomyosins with slow isomyosins and an intermediate band on pyrophosphate gels, findings similar to those in the present study with hypertrophy. A longer duration of hypertrophy may have induced more complete replacement of FM with slow, since we found the changes at 11 weeks to be greater than at 4 weeks, and histochemical fast-to-slow fibre conversion has been reported to continue for at least 21 weeks in this model (Guth \& Yellin, 1971). These findings and ours support the view that the overall activity of a muscle determines fibre characteristics and myosin phenotype, and that normal physiological stimuli of sufficient intensity can induce fibre transformation (Salmons \& Henriksson, 1981).

A well-ordered sequence of metabolic, contractile and ultrastructural alterations occurs during stimulationinduced transformation, where the changes in myosin are among the last to take place (Pette et al., 1976; Eisenberg \& Salmons, 1981; Brown et al., 1983). The isoenzyme changes we observed in the present study were still in progress at 4 weeks, even though muscle hypertrophy appeared to be complete. Thus the delayed switch of myosin isoenzymes appears also to characterize hypertrophy. The cause cannot be attributed to a deficiency in protein synthesis, which is increased within the first $24 \mathrm{~h}$ (Goldspink et al., 1983). Although gains of $20-30 \%$ in muscle weight occur within the first week (Goldberg, 1967; Jablecki \& Kaufman, 1973), this initial rapid growth appears to involve synthesis of more of the same myosin isoenzymes, in contrast with the rapid isomyosin changes with cardiac hypertrophy (Everett et al., 1984).

The hypertrophic stress was present continuously, whereas running exercise was intermittent. This may account for the lesser extent of isomyosin changes in the exercise model and the finding that the stimulus to hypertrophy overrode that of exercise when the two treatments were combined. Since the addition of exercise to the hypertrophy process induced an accumulation of SM equal to the sum of that of either treatment alone, it appears that the two stimuli were additive and may therefore exert their effects on gene transcription and protein synthesis at least in part through a similar mechanism.

At the same time, rather than representing fast-to-slow fibre transformation, as we believe occurs in response to hypertrophy, the increase in SMs and IMs in the plantaris with exercise could also represent hypertrophy of existing Type-I and -IIa fibres, at the expense of Type-IIb fibres, in response to their preferential recruitment during running exercise (Baldwin et al., 1973). The latter interpretation is supported by the persistence of all three FM isoenzymes in the exercise model and by hypertrophy of the soleus, which contains only Type-I and Type-IIa fibres, in response to running exercise. The changes in myosin isoenzymes we report here also offer insight into the molecular basis of the changes in actomyosin ATPase activity with exercise in slow and fast-red muscle observed by Baldwin et al. (1975).

The unique isoenzyme changes with hypertrophy and exercise may provide important clues regarding the functional significance of individual isoenzymes of myosin. FM1 was undetectable in hypertrophied plantaris, but remained present at control levels in exercised plantaris regardless of the magnitude of the increase in SM. This implies that FM1 has an important role in running exercise, but not in increased weight-bearing or a postural role. Similarly, the appearance of SM1 with hypertrophy and with exercise in both the plantaris and the soleus implies that SM1 may be of general importance in the adaptation of skeletal muscle to these two stimuli.

The changes in myosin isoenzymes in the slow soleus in the present study were less pronounced than those in the fast plantaris, presumably because the soleus is already well adapted for a sustained postural role and for aerobic exercise (Close, 1972). The disappearance of IM that we found in hypertrophied soleus is consistent with reports of the disappearance of histochemically identified fast-oxidative-glycolytic (Type IIa) fibres, conversion into $100 \%$ slow fibres (Guth \& Yellin, 1971; Ianuzzo et al., 1976) and with slowing of contractile properties (Lesch et al., 1968). Although hypertrophy of the soleus normally ensues after synergist removal, it does not generally occur in response to running exercise training 
(Pattengale \& Holloszy, 1972), in contrast with present findings (Table 1). These conflicting results may be attributable to the different methods used for exercise training.

We found that, on the basis of peptide-mapping analyses, the heavy-chain structure of IM is different from fast or slow heavy chains. This suggests that IM may be the isoenzyme that contains the second isoform of fast myosin heavy chain that has been reported (Starr \& Offer, 1973; Gauthier \& Lowey, 1979; Dalla Libera et al., 1980; Billeter et al., 1981; Zweig, 1981; Mabuchi et al., 1984). This second fast heavy-chain isoform, like IM, is found in Type-IIa fibres. Furthermore, this isomyosin does not appear to be a transient hybrid that only appears during muscle-type transformation, as has been suggested by others (Jolesz et al., 1981), because it is present in normal muscles.

The mixed fast and slow light-chain composition of IM that we report here agrees with that reported for IM of mouse soleus (Fitzsimons \& Hoh, 1983) and cross-innervated rat soleus (Hoh et al., 1980). As those authors pointed out, the number of alkali light-chain forms associated with IM suggests that there may be more than one species of IM co-migrating as a single band on pyrophosphate gels, some with fast and others with slow alkali light chains, or with a mixture of fast and slow light chains within a single myosin molecule. In the rabbit masseter muscle, large-diameter Type-IIa fibres contain only fast light chains, whereas smaller-diameter Type-IIa fibres contain a mixture of fast and slow light-chain types (Mabuchi et al., 1984). Thus different forms of IM may be associated with a particular subtype of Type-IIa fibre.

We conclude that unique myosin isoenzyme transitions occur in adaptations of the fast plantaris to hypertrophy and exercise. These transitions appear similar in direction and synergistic when combined. With hypertrophy, the findings indicate a fast-to-slow myosin transformation proceeding via an intermediate (IIa) myosin stage. IM, which is present in normal muscles, is shown to dominate the isoenzyme profile of both hypertrophied and exercised plantaris, but disappeared with the same stimuli in the slow soleus. Furthermore, we show that IM contains unique heavy chains, a combination of fast and slow light-chain types, and probably exists in more than one form. This evidence, along with the recent report of unique Type-IIa- and IIb-FM-heavy-chain genes in rat muscle by Izumo et al. (1986) suggests that the heavy chain of IM may be the product of a separate gene. Analysis of changes in the expression of SM-, FM-and IM-heavy-chain genes with hypertrophy would elucidate the molecular events responsible for the observed alterations in myosin phenotype.

We thank Dr. R. Z. Litten for assistance with the pyrophosphate gels. This work was supported by National Institutes of Health grant NIH AM28480.

\section{REFERENCES}

Ariano, M. A., Armstrong, R. B. \& Edgerton, V. R. (1973) J. Histochem. Cytochem. 21, 51-55

Baldwin, K. M., Reitman, J. S., Terjung, R. L., Winder, W. H. \& Holloszy, J. O. (1973) Am. J. Physiol. 225, 1045-50

Baldwin, K. M., Winder, W. W. \& Holloszy, J. O. (1975) Am. J. Physiol. 229, 422-426
Baldwin, K. M., Valdez, V., Herrick, R. E., MacIntosh, A. M. \& Roy, R. R. (1982) J. Appl. Physiol.: Respir. Environ. Exercise Physiol. 52, 467-472

Billeter, R., Heizmann, C. W., Howald, H. \& Jenny, E. (1981) Eur. J. Biochem. 116, 389-395

Brayden, J. E. \& Halpern, W. (1983) Anal. Biochem. 130, 9-13

Brown, W. E., Salmons, S. \& Whalen, R. G. (1983) J. Biol. Chem. 258, 14686-14692

Chizzonite, R. A., Everett, A. W., Clark, W. A., Jakovcic, S., Rabinowitz, M. \& Zak, R. (1982) J. Biol. Chem. 257, 2056-2065

Cleveland, D. W., Fischer, S. G., Kirschner, M. W. \& Laemmli, U. K. (1977) J. Biol. Chem. 252, 1102-1106

Close, R. I. (1972) Physiol. Rev. 52, 129-197

Dalla Libera, L., Sartore, S., Pierbon-Bormioli, S. \& Schiaffino, S. (1980) Biochem. Biophys. Res. Commun. 96, 1662-1670

Eisenberg, B. R. \& Salmons, S. (1981) Cell Tissue Res. 220, 449-471

Everett, A. W., Sinha, A. M., Umeda, P., Jakovcic, S., Rabinowitz, M., \& Zak, R. (1984) Biochemistry 23, 1596-1599

Fitzsimons, R. B. \& Hoh, J. F. Y. (1983) J. Physiol. (London) 343, 539-550

Gambke, B., Maier, A. \& Pette, D. (1985) J. Physiol. (London) 361, 34P

Gauthier, G. F. \& Lowey, S. (1979) J. Cell Biol. 81, 10-25

Giulian, G. G., Moss, R. L. \& Greaser, M. (1983) Anal. Biochem. 129, 277-287

Goldberg, A. F. (1967) Am. J. Physiol. 213, 1193-1198

Goldspink, D. F., Garlick, P. J. \& McNurlan, M. A. (1983) Biochem. J. 210, 89-98

Gollnick, P. D., Timson, B. F., Moore, R. L. \& Reidy, M. (1981) J. Appl. Physiol.: Respir. Environ. Exercise Physiol. 50, 936-943

Green, H. J., Klug, G. A., Reichmann, H., Seedorf, U., Wiehrer, W. \& Pette, D. (1984) Pflügers Arch. 400, 432-438

Gregory, P. J., Low, R. B. \& Stirewalt, W. S. (1985) J. Cell. Biol. 101, 44a

Guth, L. \& Yellin, H. (1971) Exp. Neurol. 31, 277-300

Hall-Craggs, E. C. B. (1972) J. Neurol. Sci. 15, 27-33

Hoh, J. F. Y. (1978) FEBS Lett. 90, 297-300

Hoh, J. F. Y. (1979) FEBS Lett. 98, 267-270

Hoh, J. F. Y. \& Chow, C. J. (1983) in Molecular Pathology of Nerve and Muscle (Kidman, A. D., Tomkins, J. K., Morris, C. A. \& Cooper, N. A., eds.), pp. 371-384, Humana Press, Clifton, NJ

Hoh, J. F. Y., McGrath, P. A. \& White, R. I. (1976) Biochem. J. 157, 87-95

Hoh, J. F. Y., Kwan, B. T. S., Dunlop, C. \& Kim, B. H. (1980) in Plasticity of Muscle (Pette, D., ed.), pp. 339-352, Walter de Gruyter, Berlin and New York

Hollander, M. \& Wolfe, D. (1973) Nonparametric Statistical Methods (Guba, F., Maréchal, G. \& Takács, O., eds.), pp. 114-119, Wiley, New York

Ianuzzo, C. D., Gollnick, P. D. \& Armstrong, R. B. (1976) Life Sci. 19, 1517-1524

Ianuzzo, C. D., Chen, V., Armstrong, R. B., Dabrowski, B. \& Noble, E. (1981) in Mechanism of Muscle Adaptation to Functional Requirements (Guba, F., Maréchal, G. \& Takács, O., eds.), pp. 279-290, Pergamon Press, Oxford

Izumo, S., Nadal-Ginard, B. \& Mahdavi, V. (1986) Science 231, $597-600$

Jablecki, C. \& Kaufman, S. (1973) J. Biol. Chem. 248, 1056-1062

Jolesz, F., Sreter, F. A., Mabuchi, K., Pinter, K. \& Gergely, J. (1981) in Mechanism of Muscle Adaptation to Functional Requirements (Guba, F., Maréchal, G. \& Takács, O., eds.), pp. 57-68, Pergamon Press, Oxford

Lesch, M., Parmley, W. W., Hamosh, M., Kaufman, S. \& Sonnenblick, E. H. (1968) Am. J. Physiol. 214, 685-690

Litten, R. Z., III, Martin, B. J., Low, R. B. \& Alpert, N. R. (1982) Circ. Res. 50, 856-864 
Lyons, G. E., Haselgrove, J., Kelly, A. M. \& Rubinstein, N. A. (1983) Differentiation 25, 168-175

Mabuchi, K. D., Szvetko, D., Pinter, K. \& Sreter, F. A. (1982) Am. J. Physiol. 242, C373-C381

Mabuchi, K., Pinter, K., Mabuchi, Y., Sreter, F. \& Gergely, J. (1984) Muscle Nerve 7, 431-438

Maréchal, G., Schwartz, K., Bechers-Bleukx, G. \& Ghins, E. (1984) Eur. J. Biochem. 138, 421-428

Mondon, C. E., Doklas, C. B. \& Reaven, G. M. (1980) Am. J. Physiol. 239, E169-E177

Noble, E. G., Dabrowski, L. \& Ianuzzo, C. D. (1983) Pflügers Arch. 396, 260-262

Pattengale, P. K. \& Holloszy, J. O. (1972) Am. J. Physiol. 213, 783-785

Pette, D., Müller, W., Leisner, E. \& Vrbova, G. (1976) Pflügers Arch. 364, 103-112

Pierbon-Bormioli, S., Sartore, S., Dalla Libera, L., Vitadello, M. \& Schiaffino, S. (1981) J. Histochem. Cytochem. 29, $1179-1188$

Reilly, P. J., Low, R. B. \& Stirewalt, W. S. (1984) Fed. Proc. Fed. Am. Soc. Exp. Biol. 45, 532a

Roy, R. R., Meadows, I. D., Baldwin, K. M. \& Edgerton, V. R. (1982) J. Appl. Physiol.: Respir. Environ. Exercise Physiol. 52, 473-478
Rutschmann, M., Dahlmann, B. \& Reinauer, H. (1984) Biochem. J. 221, 645-650

Salmons, S. \& Henriksson, J. (1981) Muscle Nerve 4, 94 105

Salmons, S. \& Sreter, F. A. (1976) Nature (London) 263, 30 34

Snedecor, G. W. \& Cochran, W. G. (1968) Statistical Methods, p. 114, Iowa State University Press, Ames

Sreter, F. A., Pinter, K., Jolesz, F. \& Mabuchi, K. (1982) Exp. Neurol. 75, 95-102

Starr, R. \& Offer, G. (1973) J. Mol. Biol. 81, 17-31

Thomas, J. O. (1978) Tech. Protein Enzyme Biochem. B106, $1-23$

Walsh, J. V., Jr., Burke, R. E., Rymer, W. Z. \& Tsairis, P. (1978) J. Neurophysiol. 41, 496-508

Weydert, A., Daubas, P., Caravatti, M., Minty, A., Bugaisky, G., Cohen, A., Robert, B. \& Buckingham, M. (1983) J. Biol. Chem. 258, 13867-13874

Whalen, R. G., Butler-Browne, G. S. \& Gros, F. (1978) J. Mol. Biol. 126, 415-431

Whalen, R. G., Schwartz, K., Bouveret, P., Sell, S. M. \& Gros, F. (1979) Proc. Natl. Acad. Sci. U.S.A. 76, 5197-5201

Zweig, S. E. (1981) J. Biol. Chem. 256, 11847-11853 